\title{
Are Moderate Degrees of Hyperbilirubinemia in Healthy Term Neonates Really Safe for the Brain?
}

\author{
INEKE SOORANI-LUNSING, HENK A. WOLTIL, AND MIJNA HADDERS-ALGRA
}

Department Neurology, University of Groningen, NL-9713 GZ Groningen, The Netherlands [I.S-L., M.H-A.]; Department Paediatrics, Martini Hospital, NL-9700 MM Groningen, The Netherlands [H.A.W.]

\begin{abstract}
ABSTR
In 1994 the American Academy of Pediatrics recommended
more liberal rules for the treatment of hyperbilirubinemia in
healthy term newborns. Yet, the safety of moderate degrees of
hyperbilirubinemia in healthy term newborns is debated. To
evaluate the safety of moderate degrees of hyperbilirubinemia,
we assessed neurologic condition of 20 healthy nonhemolytic
term newborns with peak total serum bilirubin levels of $233-444$
$\mu$ mol/L and 20 control infants matched for sex and gestational
age at birth. Neurologic condition was evaluated with techniques
focusing on the presence of minor neurologic dysfunction: in the
newborn period according to Prechtl, at 3 mo on the basis of the
quality of general movements, and at 12 mo according to Tou-
\end{abstract}
In 1994 the American Academy of Pediatrics recommended new guidelines for the treatment of hyperbilirubinemia in healthy term newborns without hemolytic disease (1). Before 1994 treatment was started generally when total serum bilirubin levels exceeded 239-257 $\mu \mathrm{mol} / \mathrm{L}$ (14-15 mg/dL) (2). According to the new, more liberal guidelines it is acceptable to postpone in infants aged at least $72 \mathrm{~h}$ the onset of treatment until the total serum bilirubin level exceeds $340 \mu \mathrm{mol} / \mathrm{L}$. The new recommendations were also implemented in clinical practice in the Netherlands (3).

The rationale for the new recommendations was that therapeutic interventions for hyperbilirubinemia in healthy term infants may carry significant risk relative to the uncertain risk of hyperbilirubinemia in this population. The uncertain risk was considered to be relatively low. This point of view was supported by the review of Newman and Maisels, which concluded that bilirubin levels below $340 \mu \mathrm{mol} / \mathrm{L}$ did not have a significantly adverse effect on neurologic, mental (IQ) and hearing development (4). Yet, at present it is not clear whether moderate degrees of hyperbilirubinemia (205-340 $\mu \mathrm{mol} / \mathrm{L})(2)$

Received February 10, 2000; Accepted August 8, 2000

Correspondence: Dr. Mijna Hadders-Algra, Developmental Neurology, University Hospital Groningen, Hanzeplein 1, NL-9713 GZ Groningen, The Netherlands; e-mail: m.hadders-algra@med.rug.nl wen. Moderate hyperbilirubinemia turned out to be associated with a significant increase in minor neurologic dysfunction throughout the first year of life. At 12 mo a strong dose-response relationship between the degree of hyperbilirubinemia and the severity of minor neurologic dysfunction was present. Our results indicate that total serum bilirubin levels $335 \mu \mathrm{mol} / \mathrm{L}$ should be avoided. (Pediatr Res 50: 701-705, 2001)

\section{Abbreviations \\ CRIB, clinical risk index of babies IQ, intelligence quotient}

in term infants do not result in an increased risk for so-called minor developmental disorders, such as clumsiness and attention deficit hyperactivity disorder. In this respect it is good to realize that the term minor in minor developmental disorders is more related to the degree of dysfunction of the neural substrate than to its clinical impact, as minor developmental disorders often strongly interfere with the child's activities of daily life $(5,6)$. In fact, the data of the American Collaborative Perinatal Project, in which a large number of children born in the sixties were followed extensively, suggest that moderate degrees of hyperbilirubinemia are associated with an increased risk of minor motor problems at school-age (7).

The aim of the present study is to investigate whether moderate degrees of hyperbilirubinemia are safe with regard to the development of minor neurologic dysfunction. To this end two groups of twenty otherwise healthy term newborns, one with a moderate hyperbilirubinemia and a matched control group were assessed neurologically in the newborn period, and at the ages of three and $12 \mathrm{mo}$. The neurologic assessment techniques we used were specially designed to detect minor neurologic dysfunction. In addition, information on behavior was collected at the age of $12 \mathrm{mo}$. The following hypotheses were tested: Moderate hyperbilirubinemia in term healthy infants does not result in an increase of a) minor neurologic dysfunction at $3 \mathrm{mo}, \mathrm{b}$ ) minor neurologic dysfunction dysfunc- 
tion at $12 \mathrm{mo}, \mathrm{c})$ behavioral problems, such as attention problems at $12 \mathrm{mo}$.

\section{METHODS}

Participants. The study group consisted of twenty healthy term Caucasian infants born at the Martini Hospital in Groningen between November 1st 1997 and June 30th 1998, whose highest serum total bilirubin level had been $>220$ $\mu \mathrm{mol} / \mathrm{L}$ (range $233-444 \mu \mathrm{mol} / \mathrm{L}$ ). A control group was formed of twenty healthy Caucasian infants without hyperbilirubinemia, born in the same period and matched for sex and gestational age at birth to the study group (Table 1). Bilirubin levels in the control group were not available as it was considered ethically unjustified to submit healthy nonjaundiced infants to vena puncture just to confirm their physiologic bilirubin status. In the matching with respect to gestational age at birth we accepted a difference of maximally one week. All children were born at a gestational age of at least 36 completed weeks of pregnancy, had a birthweight above the tenth centile of the growthcurve (8) and had Apgar scores at 5 min of 9 nine or more. Children were excluded when one or more of the following conditions was present: congenital anomaly, hemolytic disease, sepsis, meningitis or metabolic derangement. All infants scored zero at the Clinical Risk Index of Babies (CRIBscore) (9). Ten of the infants with hyperbilirubinemia were treated with phototherapy. Phototherapy was given in accordance to the national guidelines on the prevention of neonatal bilirubin neurotoxicity, which state that infants with a total serum bilirubin level of $310 \mu \mathrm{mol} / \mathrm{L}$ on the second day of life or with a total serum bilirubin level of $340 \mu \mathrm{mol} / \mathrm{L}$ from the third day of life onwards, should be given this treatment (3). The peak of the total serum bilirubin level in the infants who received phototherapy was significantly higher than that of the ten infants who did not get phototherapy (Table 1). The studyand control group turned out to be comparable with respect to

Table 1. Perinatal and neonatal condition and social status in study and control group

\begin{tabular}{|c|c|c|}
\hline & Study group & Control group \\
\hline Peak TSB in $\mu$ mol $/ 1$ (mean \pm SD) & $313 \pm 62$ & $\begin{array}{l}\text { No data available } \\
\text { (no jaundice) }\end{array}$ \\
\hline No phototherapy $(\mathrm{n}=10)$ & $284 \pm 41$ & \\
\hline With phototherapy $(\mathrm{n}=10)$ & $342 \pm 67^{*}$ & \\
\hline $\operatorname{Sex}(M: F)$ & $15: 5$ & $15: 5$ \\
\hline $\begin{array}{l}\text { Gestational age at birth in days (mean } \pm \\
\text { SD) }\end{array}$ & $272 \pm 12$ & $278 \pm 12$ \\
\hline Number of infants aged $36 \mathrm{wk}$ at birth & 4 & 2 \\
\hline Birthweight in $\mathrm{g}$ (mean $\pm \mathrm{SD})$ & $3480 \pm 403$ & $3357 \pm 402$ \\
\hline Rate of cesarean section & $2 / 20$ & $14 / 20 \dagger$ \\
\hline Neonatal breast feeding & $15 / 20$ & $14 / 20$ \\
\hline \multicolumn{3}{|l|}{ Social class $\nleftarrow$} \\
\hline Low & $8(40 \%)$ & $4(20 \%)$ \\
\hline Middle & $6(30 \%)$ & $13(65 \%)$ \\
\hline High & $6(30 \%)$ & $3(15 \%)$ \\
\hline
\end{tabular}

$\mathrm{TSB}=$ total serum bilirubin; wk $=$ weeks.

* phototherapy - or $+: t$-test, $p=0.03$.

$\dagger$ Study vs. control group: $\chi^{2}=12.6, p<0.001$.

$\ddagger$ Social class according to maternal education (low = primary education/ junior vocational training; middle $=$ secondary general education/senior vocational training; high $=$ university education/vocational colleges (33). pre-, peri- and neonatal condition, except for the presence of hyperbilirubinemia and the rate of Caesarean section. The latter was significantly higher in the control group than in the study group (Table 1). The different rate of Caesarian section in the groups is related to the Dutch system of obstetrical care, in which a high proportion of healthy infants is born at home. Infants born by means of an instrumental delivery, including Caesarean section, are born in the hospital. All parents gave informed consent and the procedures were approved by the Medical Ethics Committee of the Martini Hospital.

Procedures. To document neurologic status in the newborn period all infants were assessed at the age of 3 to $8 \mathrm{~d}$ with the standardized technique of Prechtl (10). A similar type of standardized neurologic examination was carried out at the age of $12 \mathrm{mo}$ (11). According to these neurologic examinations children were classified as neurologically normal, minor neurologic dysfunction or definitely abnormal (12). Children were classified as definitely abnormal in case of the presence of clear neurologic syndromes, such as a marked hypertonia, a marked hypotonia, marked asymmetries or (in newborns) a hyperexcitability syndrome. They were classified as minor neurologic dysfunction when they showed milder forms of a clear neurologic syndrome. Examples are mild abnormalities in postural behavior, the presence of high frequency tremors, mild deviancies in muscle tone regulation and mild asymmetries in infantile reactions and tendon reflexes. In analogy to classifications at older ages, the classification of minor neurologic dysfunction at the age of 12 mo was split up into two subclassifications: minor neurologic dysfunction type 1 and minor neurologic dysfunction type 2. Minor neurologic dysfunction type 1 denotes a simple form of minor neurologic dysfunction and can be regarded as a normal, but nonoptimal condition of the nervous system, whereas minor neurologic dysfunction type 2 represents a more complex form of minor neurologic dysfunction, which is more clearly related to early brain damage $(13,14)$. At early age, i.e., before the onset of puberty, the difference between the two forms of minor dysfunction is aspecific. It simply consists of a difference in the number of dysfunctions present. But beyond puberty the difference is specific, with type 1 dysfunctions indicating the presence of mild muscle tone dysregulation or choreiform dyskinesia and type 2 dysfunctions the presence of mild coordination problems or fine manipulative disability (14). In the present study, minor neurologic dysfunction type 1 at the age of $12 \mathrm{mo}$ denoted the presence of hypotonia without significant reflex or postural deviancies, and minor neurologic dysfunction type 2 mild abnormalities in muscle tone regulation in combination with significant postural and reflex dysfunction. The two neurologic assessments were performed by ISL, who occasionally knew group membership of the children, but who was never aware of the degree of hyperbilirubinemia of the study children.

At 3 mo neurologic condition was evaluated by means of an assessment of the quality of General Movements. The assessment of general movements is a newly developed sensitive tool to evaluate brain function in young infants (15-17). A video recording of 20 min was made of spontaneous motility in supine position while the infant was in an alert, active, non- 
crying behavioral state. The video-recordings were 'blindly' assessed by ISL and MHA. The quality of general movements was classified as normal (perfectly complex, variable and fluent General movements), mildly abnormal (moderately complex and variable movements, which are not fluent) and definitely abnormal (lack of complexity, variation and fluency (16)). In the large majority of cases the two observers agreed upon the classification of the quality of general movements, which is in line with previously reported high values of interscorer agreement (Kappa-values $>0.80)(16,17)$. In the few instances of disagreement, results were discussed until agreement was reached.

In the jaundiced infants total and direct reacting bilirubin measurements were performed with a Beckman Coulter Analyzer (Beckman Inc., Fullerton, CA, USA). The course of the hyperbilirubinemia was recorded. In all infants data on the obstetrical and neonatal condition, including the CRIB score (9) and on intercurrent diseases during the first year of life were documented on standardized forms. Behavior was evaluated at the age of 12 mo by means of a parental questionnaire. The format of the questions was: "All children are sometimes ...; do you regard your child as more ..., less ..., or don't you see any difference?". The behavioral characteristics evaluated in this way consisted of 'hyperactive', 'distractible', 'poorly concentrated', 'irritable', 'stubborn', 'having temper tantrums', 'easy to handle', 'cheerful', 'easily frightened', 'shy', and 'anxious' $(6,18)$.

The differences in the rates of neurologic and behavioral problems between the study and control group were evaluated with nonparametric tests, such as the Fisher Exact Test and $\chi^{2}$ test. Logistic regression analysis was used to evaluate the contribution of possibly confounding factors, such as sex, gestational age at birth, birth weight, presence or absence of Caesarian section, social class, presence or absence of neonatal breast feeding and the presence or absence of intercurrent diseases on neurologic and behavioral development. Logistic regression analysis involved testing of various models and selection of the model which was significant and explained the highest degree of variance. Throughout the analyses, differences with a $p$-value $\leqslant 0.05$ were considered to be statistically significant (two-tailed testing).

\section{RESULTS}

In the newborn period, none of the infants showed a definitely abnormal neurologic condition. But the rate of minor neurologic dysfunction was significantly higher in the study group (14 out of 20) than in the control group (5 out of 20; $X^{2}$ $=6.4, p<0.05)$. Note that the rate of dysfunction in the control group was in agreement with previous reports on the prevalence of minor neurologic dysfunction in the general population $(13,17)$. Logistic regression analysis revealed that the effect of hyperbilirubinemia on neonatal neurologic condition remained significant. The best model explaining minor neurologic dysfunction in the newborn period consisted of the combination of birthweight (a nonsignificant contribution of lower birthweight) and study- versus control group member- ship. The odds of developing neonatal minor neurologic dysfunction was 6.62 (95\% confidence intervals 1.59 to 27.51$)$.

At the age of 3 mo more or less similar results were obtained. None of the infants showed definitely abnormal general movements. But again the rate of minor neurologic dysfunction, in the form of mildly abnormal general movements, was higher in the study (11 out of 20) than in the control group ( 5 out of $\left.20 ; \mathrm{X}^{2}=3.84, p=0.05\right)$. Logistic regression analysis confirmed the effect of hyperbilirubinemia on the quality of general movements at $3 \mathrm{mo}$. Like in the neonatal period the best model explaining minor neurologic dysfunction consisted of birthweight (again a nonsignificant contribution for lower birthweight) and group membership. The odds of developing mildly abnormal general movements was 3.75 (95\% confidence intervals 1.03 to 14.53 ).

At the third assessment, at the age of 12 mo neurologic results were similar again. None of the children showed frank neurologic pathology. But the rate of minor neurologic dysfunction was significantly higher in the study group (10 out of 20 ) than in the control group ( 2 out of $20 ; \mathrm{X}^{2}=5.8, p<0.05$ ). The difference in the rate of minor neurologic dysfunction was mainly due to a difference in the rate of the type 2 dysfunction (study group 5 out of 20, control group 0 out of 20; Fisher, $p$ $=0.02)$. Logistic regression analysis showed that the model explaining minor neurologic dysfunction (minor neurologic dysfunction type 1 and type 2) best consisted of the sex of the child (with a nonsignificant disadvantage for boys) and group membership. The odds of developing minor neurologic dysfunction at 12 mo was 9.47 (95\% confidence intervals 1.67 53.65). Neurologic outcome at 12 mo was strongly related to the height of the peak of the total serum bilirubin. All five children with minor neurologic dysfunction type 2 had had total serum bilirubin levels of $335 \mu \mathrm{mol} / \mathrm{L}$ or higher (Fig. 1). Four of them had received phototherapy and one did not (the peak of the total serum bilirubin of this infant was $355 \mu \mathrm{mol} /$ L). The height of the peak of the total serum bilirubin level was related to the duration of the elevated total serum bilirubin level $(>340 \mu \mathrm{mol} / \mathrm{L} ; \mathrm{r}=0.83, p<0.001)$. Yet, logistic

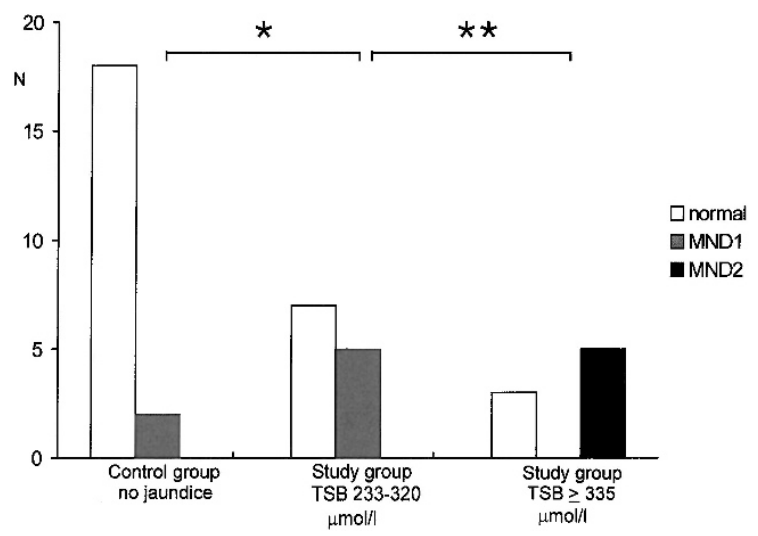

Figure 1. Neurologic condition at 12 mo of age in the control group, the study infants with a moderately high peak of the total serum bilirubin (233-320 $\mu \mathrm{mol} / \mathrm{L})$ and the study infants with a high peak of the total serum bilirubin (335-444 $\mu \mathrm{mol} / \mathrm{L})$. *Proportion of minor neurologic dysfunction (MND) type 1, Fisher: $p=0.04, * *$ proportion of minor neurologic dysfunction type 2 , Fisher: $p<0.01$. 
regression analysis showed that it was the height of the peak which was most closely related to neurologic outcome and not the number of days during which the total serum bilirubin level was elevated. The height of the peak of the total serum bilirubin was not related to the neurologic condition at younger ages.

According to the parental questionnaires, the behavior of the study and control children at the age of 12 mo did not differ.

\section{DISCUSSION}

The present study shows that moderate degrees of hyperbilirubinemia are associated with an increase in minor neurologic dysfunction throughout the first year of life. The multivariate analyses indicated that the increase in neurologic dysfunction in the group of hyperbillirubinaemic infants could not be explained by their nonsignificantly younger gestational age at birth. Moreover, our study demonstrated that neurologic outcome became more strongly related to the height of the peak of the total serum bilirubin with increasing age, i.e. with increasing differentiation of the CNS. Total serum bilirubin levels 335 $\mu \mathrm{mol} / \mathrm{L}$ were clearly associated with mild abnormalities in muscle tone regulation in combination with significant postural and reflex dysfunction at the age of 12 mo. These neurologic abnormalities can be regarded as minor forms of chronic bilirubin encephalopathy (19). Bilirubin encephalopathy is caused by neuronal injury in subcortical areas, such as the basal ganglia, the dentate nucleus of the cerebellum and various nuclei in the brain stem $(19,20)$. Likewise, the higher prevalence of mildly abnormal general movements at the age of three months in the study group than in the control group, supports the notion that moderate degrees of hyperbilirubinemia interfere with a normal function of the monoaminergic systems $(16,17)$. A recent study indicated that the presence of mildly abnormal general movements at the age of three months is significantly related to the development of minor neurologic dysfunction, attention problems and aggressive behavior at school age (17). Yet, in the present study we were not able to find significant differences in behavior between the children with neonatal hyperbilirubinemia and those without, which might be due to the young age at which we evaluated the children's behavior.

Our data point in the same direction as the data of Newman and Klebanoff (7) and those of Grimmer and coworkers (21). Newman and Klebanoff, who analyzed data of the large population of the American Collaborative Perinatal Project, reported that total serum bilirubin-levels exceeding $342 \mu \mathrm{mol} / \mathrm{L}$ were associated with an increase of minor motor problems, such as a mild hypotonia, awkwardness or nonspecific gait abnormalities, at the age of 7 y (7). Grimmer et al. who studied 16 children with neonatal total serum bilirubin levels of $340-$ $510 \mu \mathrm{mol} / \mathrm{L}$ and 18 case controls between the ages of 5-15 y of age, found that the children with moderate neonatal hyperbilirubinemia showed a significantly higher prevalence of choreiform dyskinesia (21). Unlike Newman and Klebanoff we do not discard the finding of an excess of minor neurologic dysfunction in children with moderate hyperbilirubinemia as clinically irrelevant (7). On the contrary, we know too well that children with minor neurologic dysfunction, and especially those with minor neurologic dysfunction type 2 , are at high risk for behavioral and learning problems $(5,6,14,18)$. Of course, the long term consequences of moderate hyperbilirubinemia for motor, cognitive and behavioral development should be investigated in follow-up at school age.

The dose-response relationship between the degree of hyperbilirubinemia and the degree of neurologic dysfunction at 12 mo suggests a causal relationship between the two. Our study suggests that especially total serum bilirubin levels $\geqslant$ $335 \mu \mathrm{mol} / \mathrm{L}$ should be avoided. How can this be accomplished? In the first place by adequate monitoring of total serum bilirubin (22) and providing an optimal calory intake (23). Secondly, by lowering bilirubin levels which are getting in the danger-zone. The primary choice for treatment is phototherapy, as phototherapy does lower bilirubin level $(23,24)$ and does reduce the risk of exchange transfusion (25). Moreover, phototherapy can be given at home and only minimally interferes with maternal nursing routines (26). Side effects of phototherapy, such as tanning, the bronze baby syndrome, diarrhea, lactose intolerance, hemolysis, dehydration and skin rashes occur rarely and transiently (27). In addition, phototherapy can induce transient hemodynamic changes, but these are not accompanied by significant changes in the cerebral circulation $(28,29)$. A study, which used brain-stem auditory responses, showed that phototherapy has a positive effect on the acute neurologic condition of the infant (30). Yet, a positive effect of phototherapy on long-term development has never been demonstrated. Seidman et al. who evaluated IQ at the age of $17 \mathrm{y}$ in subjects born before and after the introduction of phototherapy in 1971, concluded that phototherapy had no independent effect on IQ scores (31). Similar conclusions were drawn by Scheidt and coworkers (32). The latter group carried out a neurologic, psychometrical, behavioral and auditory evaluation at the ages of 1 and $6 \mathrm{y}$ in children who participated in a randomized controlled trial on the effect of phototherapy. According to the study's protocol the infants were randomly assigned to the phototherapy or control group at predefined bilirubin levels. At 1 and 6 y the children who received neonatal phototherapy performed equally well on all outcome measures as the nontreated children. But, the rate of exchange transfusions, which were carried out at predetermined bilirubin values, was significantly higher in the control infants than in the infants with phototherapy. The study confirmed that phototherapy is an effective instrument in lowering serum bilirubin levels and indicated that phototherapy it is not associated with an excess or a decrease in long-term morbidity. In addition, it demonstrated the effectiveness of active evaluation and treatment of hyperbilirubinemia.

\section{CONCLUSION}

Moderate hyperbilirubinemia $(233-444 \mu \mathrm{mol} / \mathrm{L})$ is associated with an increase in minor neurologic dysfunction throughout the first year of life. The dose-response relationship between the degree of hyperbilirubinemia and the severity of the minor neurologic dysfunction condition indicates that total serum bilirubin levels $\geqslant 335 \mu \mathrm{mol} / \mathrm{L}$ should be avoided. 
Acknowledgments. We thank the families whose participation made the project possible and Dr. E. Oldekamp for assistance with the figures.

\section{REFERENCES}

1. Provisional Committee for Quality Improvement and Subcommittee on Hyperbilirubinemia 1994 Practice parameter: management of hyperbilirubinemia in the healthy term newborn. Pediatrics 94:558-565

2. Newman TB, Maisels MJ 1992 Evaluation and treatment of jaundice in the term newborn: a kinder, gentler approach. Pediatrics 89:809-818

3. Fetter WPF, Van de Bor M, Brand PLP, Kollée LAA, De Leeuw R, De Nef JJE 1997 Hyperbilirubinemie bij gezonde voldragen pasgeborenen; richtlijnen voor diagnostiek en behandeling. Ned Tijdschr Geneeskd 141:140-143

4. Newman TB, Maisels MJ 1990 Does hyperbilirubinemia damage the brain of healthy full-term infants? Clin Perinatol 17:331-357

5. Gillberg IC, Gillberg C 1989 Children with preschool minor neurodevelopmental disorders. IV: Behaviour and school achievement at age 13. Dev Med Child Neurol $31: 3-13$

6. Soorani-Lunsing RJ, Hadders-Algra M, Huisjes HJ, Touwen BCL 1994 Neurobehavioural relationships after the onset of puberty. Dev Med Child Neurol 36:334-343

7. Newman TB, Klebanoff MA 1993 Neonatal hyperbilirubinemia and long-term outcome: another look at the collaborative perinatal project. Pediatrics 92:651-657

8. Kloosterman GJ 1970 On intrauterine growth. Int J Gynaecol Obstet 8:895-912

9. International Neonatal Network 1993 The CRIB (clinical risk index for babies) score: a tool for assessing initial neonatal risk and comparing the performance of neonatal intensive care units. Lancet 342:193-198

10. Prechtl HFR 1977 The neurological examination of the full term newborn infant, 2nd ed. Clinics in developmental medicine, No. 63. Heinemann, London, pp 68

11. Touwen BCL 1976 Neurological development in infancy. Clinics in developmental medicine, No. 58. Heinemann, London, pp 150

12. Bierman-van Eendenburg MEC, Jurgens-van der Zee AD, Olinga AA, Huisjes HJ, Touwen BCL 1981 Predictive value of neonatal neurological examination: a follow-up study at 18 months. Dev Med Child Neurol 23:296-305

13. Hadders-Algra M, Huisjes HJ, Touwen BCL 1988 Perinatal correlates of major and minor neurological dysfunction at school age: a multivariate analysis. Dev Med Child Neurol 30:472-481

14. Hadders-Algra M, Touwen BCL 2001 Perinatal events and soft neurological signs in neurobehavioral outcome. Dev Neuropsychol (in press)

15. Prechtl HFR, Einspieler C, Cioni G, Bos A, Ferrari F, Sontheimer D 1997 An early marker of developing neurological handicap after perinatal brain lesions. Lancet 339:1361-1363
16. Hadders-Algra M, Klip-Van den Nieuwendijk, Martijn A, van Eykern LA 1997 Assessment of general movements: towards a better understanding of a sensitive method to evaluate brain function in young infants. Dev Med Child Neurol 39:89-99

17. Hadders-Algra M, Groothuis AMC 1999 Quality of general movements in infancy is related to neurological dysfunction, ADHD, and aggressive behaviour. Dev Med Child Neurol 41:381-391

18. Hadders-Algra M, Huisjes HJ, Touwen BCL 1988 Perinatal risk factors and minor neurological dysfunction: significance for behaviour and school achievement at nine years. Dev Med Child Neurol 30:482-491

19. Connolly AM, Volpe JJ 1990 Clinical features of bilirubin encephalopathy. Clin Perinatol 17:371-379

20. Turkel SB 1990 Autopsy findings associated with neonatal hyperbilirubinemia. Clin Perinatol 2:381-394

21. Grimmer I, Berger-Jones K, Bührer C, Brandl U, Obladen M 1999 Late neurological sequela of non-hemolytic hyperbilirubinemia of healthy term neonates. Acta Paediatr 88:661-663

22. Maisels MJ, Newman TB 1999 Predicting hyperbilirubinemia in newborns: the importance of timing. Pediatrics 103:493-495

23. Wu PYK, Hodgman JE, Kirkpatrick BV, White NB, Phil M, Bryla DA 1985 Metabolic aspects of phototherapy. Pediatrics 75(suppl):427-433

24. Martinez JC, Maisels J, Otheguy L, Garcia H, Savorani M, Mogni B, Martinez JC 1993 Hyperbilirubinemia in the breast-fed newborn: a controlled trial of four interventions. Pediatrics 91:470-473

25. Valaes T, Koliopoulos T, Koltsidopoulos A 1996 The impact of phototherapy in the management of neonatal hyperbilirubinemia: a comparison of historical cohorts. Acta Paediatr 85:273-276

26. Meropol SB, Luberti AA, De Jong AR, Weiss JC 1993 Home phototherapy: use and attitudes among community pediatricians. Pediatrics 91:97-99

27. Poland RL, Ostrea EM 1986 Neonatal hyperbilirubinemia. In: Klaus MH, Fanaroff AA (eds) Care of the high-risk neonate. WB Saunders, Philadelphia, pp. 239-261

28. Benders MJ, Van Bel F, Van de Bor M 1999 Haemodynamic consequences of phototherapy in term infants. Eur J Pediatr 158:323-328

29. Amato M, Donati F 1991 Cerebral blood flow velocity in term infants treated with phototherapy. Brain Dev 13:417-419

30. Tan KL, Skurr BA, Yip YY 1992 Phototherapy and the brain-stem auditory evoked response in neonatal hyperbilirubinemia. J Pediatr 120:306-308

31. Seidman DS, Paz I, Stevenson DK, Laor A, Danon YL, Gale R 1991 The effect of phototherapy for neonatal hyperbilirubinemia on long term cognitive performance. Pediatr Res 29:265A

32. Scheidt PC, Bryla DA, Nelson KB, Hirtz DG, Hoffman HJ 1990 Phototherapy for neonatal hyperbilirubinemia: six year follow-up of the national institute of child health and human development clinical trial. Pediatrics 85:455-463

33. Herngreen WP, Reerink JD, Van Noord-Zaadstra BM, Verloove-Vanhorick SP, Ruys JH 1992 The SMOCC-Study: design of a representative cohort of live-born infants in the Netherlands. Eur J Publ Health 2:117-122 